\section{References}

1. Coppin JD, et al. Increased time spent on terminal cleaning of patient rooms may not improve disinfection of high-touch surfaces. Infect Control Hosp Epidemiol 2019;40:605-606.

2. Hooker EA. Increased time spent on terminal cleaning of patient rooms may not improve disinfection of high-touch surfaces. Infect Control Hosp Epidemiol 2019;40:1086.

3. Huslage K, Rutala WA, Sickbert-Bennett E, Weber DJ. A quantitative approach to defining "high-touch" surfaces in hospitals. Infect Control Hosp Epidemiol 2010;31:850-853.
4. Jinadatha C, Villamaria FC, Ganachari-Mallappa N, et al. Can pulsed xenon ultraviolet light systems disinfect aerobic bacteria in the absence of manual disinfection? Am J Infect Control 2015;43:415-417.

5. O'Hara RB, Kotze DJ. Do not log-transform count data. Methods Ecol Evol 2010;1:118-122.

6. Amrhein V, Greenland S, McShane B. Retire statistical significance. Nature 2019; 567:305-307.

7. Harrington, D. et al. New guidelines for statistical reporting in the journal. N Engl J Med 2019; 381:285-286.

\title{
Impact of peer comparison on carbapenem use among inpatient prescribers at a community hospital
}

\author{
Rossana M. Rosa MD ${ }^{1}$ and Amanda M. Bushman PharmD² \\ ${ }^{1}$ Infectious Diseases Service, UnityPoint Health-Des Moines, Des Moines, lowa and ${ }^{2}$ Department of Pharmacy, UnityPoint Health-Des Moines, Des Moines, lowa
}

To the Editor-The Infectious Diseases Society of America (IDSA) guidelines for the implementation of an antimicrobial stewardship program (ASP) recommend using preauthorization, prospective review, and feedback or a combination of these as core strategies for ASPs. ${ }^{1}$ Behavioral interventions, such as peer comparison, are included among the Core Elements of Outpatient Antimicrobial Stewardship developed by the Centers for Diseases Control and Prevention (CDC). ${ }^{2}$ However, evidence on the use of peer comparison by inpatient ASPs is limited.

To further understand the applicability of a behavioral intervention in this setting, we conducted an intervention consisting of peer comparison of the quantitative and qualitative use of carbapenems among inpatient prescribers. The main outcome of interest was carbapenem days of therapy (DOT) per 1,000 patient days. This study was conducted at a 374-bed hospital and its level 1 trauma center in Des Moines, Iowa. The preintervention period was December 1, 2016, to November 30, 2017, and the postintervention period was December 1, 2017, to November 30, 2018.

The intervention was limited to internal medicine and surgery house staff, and the hospitalists, critical care specialists, and surgeons directly working with house staff. These 5 groups of "peers" were used for direct comparisons. By targeting these groups we estimated that we would reach $>80 \%$ of the prescribers of carbapenems at our facility.

Each DOT prescribed was reviewed and its appropriateness was determined based on previously published definitions. ${ }^{3}$ Each DOT was assigned to the physician considered to be directly responsible for the patient's care that day, which was determined by authorship of progress notes. In cases in which house staff authored the progress note, both attending and trainee were assigned the DOT. The component of the peer comparison report concerning quantitative use was calculated by adding the number of DOTs

Author for correspondence: Rossana M. Rosa MD, E-mail: rossana.m.rosa@gmail. com$^{*}$ PREVIOUS PRESENTATION: The results from this study were presented at ID Week 2019, on October 4, 2019, in Washington, DC.

Cite this article: Rosa RM and Bushman AM. (2020). Impact of peer comparison on carbapenem use among inpatient prescribers at a community hospital. Infection Control \& Hospital Epidemiology, 41: 376-378, https://doi.org/10.1017/ice.2019.377 adjudicated to each physician. The component indicating appropriateness of use was calculated using the following formula:

Percentage of appropriate use by physician

= Total no. of appropriate DOT by physician/

Total no. of DOT by physician

A similar calculation was done for each peer group using the following formula:

$$
\begin{aligned}
& \text { Percentage of appropriate use by peer group } \\
& =\text { Total no. of appropriate DOT by peer group/ } \\
& \text { Total no. of DOT by peer group }
\end{aligned}
$$

Reports on quantity and appropriateness by each individual and in comparison with their peers were sent by e-mail on a monthly basis (Supplemental Material online).

An interrupted time series analysis was preferred to determine changes in the slope of rate of hospital-level carbapenem DOT per 1,000 patient days following onset of intervention. The impact of the intervention was modeled as a gradual change in the trend of carbapenem use, and a Poisson regression model was used. Data were assessed for autocorrelation and none was found. No other interventions targeting carbapenem use were implemented during this study period.

During the 12 months of the intervention, an average of 24 e-mails per month were sent and a total of 91 physicians were contacted. The average carbapenem DOT per 1,000 patient days was 15.6 in the preintervention period and 15.2 in the postintervention period.

Following onset of the intervention, no change in the trend of carbapenem use was observed (incidence rate ratio [IRR], 1.04; $95 \%$ confidence interval $[\mathrm{CI}], 0.98-1.10 ; P=.21$ ) (Fig. 1a). The impact of the intervention was also analyzed by medical and surgical services, and trends of carbapenem use also remained stable. In the medical service the IRR was 0.98 (95\% CI, 0.92-1.05; $P=.612$ ) (Fig. 1b), and in the surgical service, the IRR was 1.05 (95\% CI, 0.99-1.13; $P=.11$ ) (Fig. 1c). The percentage of 


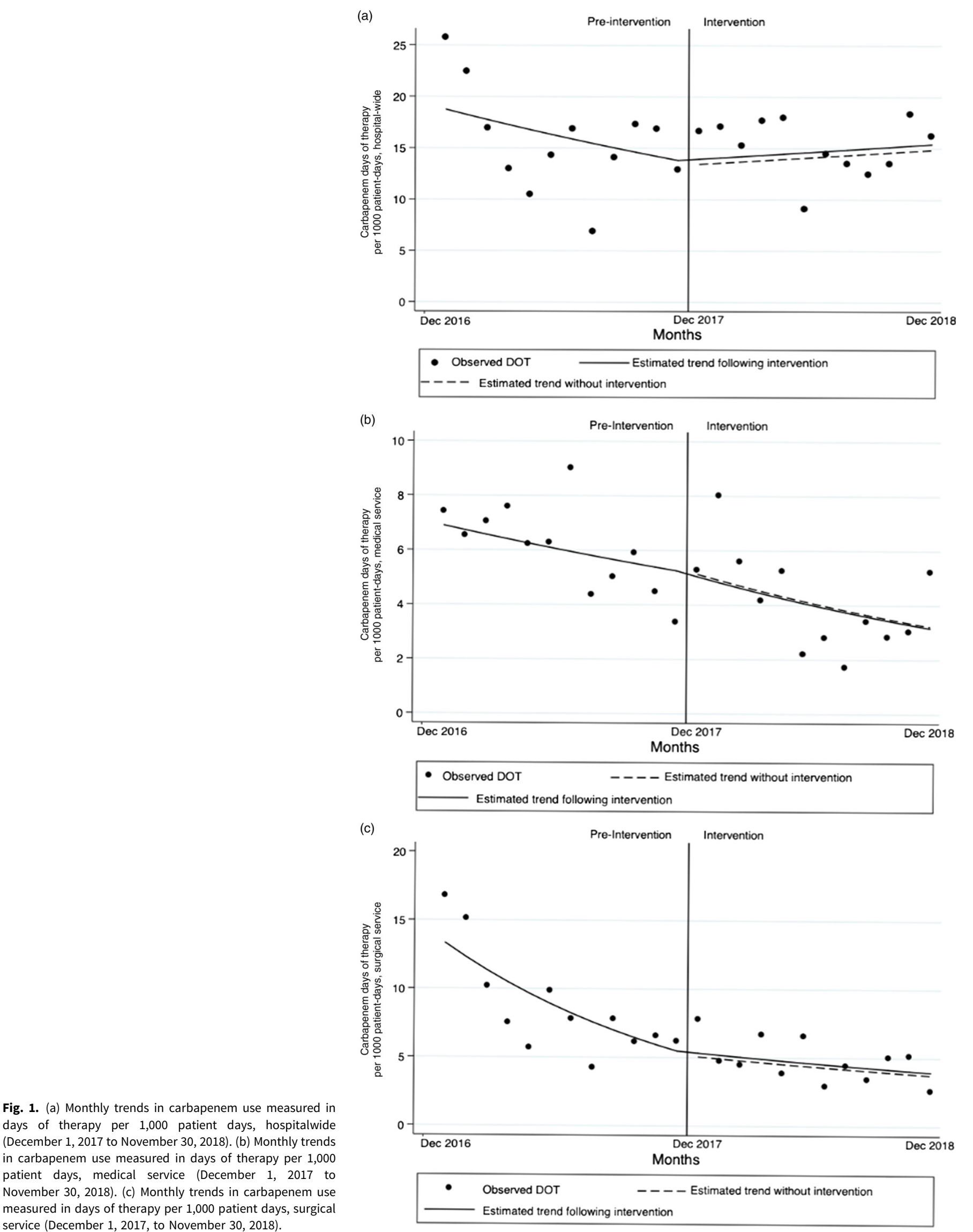


appropriate use remained constant across the different prescribing groups during the study period (Supplementary Fig. 1 online).

In this study, implementing peer comparison had no impact on the appropriateness or overall rates of carbapenem use among hospitalized patients. Our study highlights the complexities of implementing this strategy on inpatient settings. The use of peer comparison has been shown to decrease antibiotic prescribing in outpatient settings ${ }^{4,5}$; however, prescribing dynamics are different in inpatient settings, where the physician who initially prescribes an antibiotic may not be responsible for patient care in subsequent days and $>1$ medical service may be involved in management. This setting poses a challenge from accountability and logistic perspectives.

The baseline use of the antibiotic class targeted could influence the results of this type of intervention. Allen et al. ${ }^{6}$ conducted a quasiexperimental before-and-after study targeting high-volume fluoroquinolone prescribers across 16 community hospitals in Florida. All hospitals had ASPs. Individuals belonging to 3 cohorts (ie, internal medicine, family medicine, and hospitalists; intensivists; and infectious diseases specialists) were provided with individual and facility-specific peer cohort percentage of total antibiotic days of therapy attributable to fluoroquinolones, as well as education on decreasing fluoroquinolone use. Fluoroquinolone use declined by $29 \%$ in the postintervention period, with larger changes seen in facilities with the highest baseline use. In contrast to this study, our intervention targeted a different antibiotic class with low baseline use.

The limitations of our study include its observational design and that it was conducted at a single center. Furthermore, our intervention did not include all hospital providers prescribing carbapenems. However, we included the physician groups responsible for the majority of carbapenem DOTs at our institution.

In conclusion, we found no impact on trends of carbapenem use following the addition of peer comparison to a well-established inpatient ASP using prospective review and feedback as its main strategy. Future studies can help determine whether this strategy could be applied to other antibiotics classes or specific syndromes among hospitalized patients.

Supplementary material. To view supplementary material for this article, please visit https://doi.org/10.1017/ice.2019.377

Acknowledgments. None.

Financial support. No financial support was provided relevant to this article.

Conflicts of interest. All authors report no conflicts of interest relevant to this article.

\section{References}

1. Barlam TF, Cosgrove SE, Abbo LM, et al. Implementing an antibiotic stewardship program: guidelines by the Infectious Diseases Society of America and the Society for Healthcare Epidemiology of America. Clin Infect Dis 2016;62:e51-e77.

2. The Core Elements of Outpatient Antibiotic Stewardship. Centers for Disease Control and Prevention website. https://www.cdc.gov/antibioticuse/community/pdfs/16_268900-A_CoreElementsOutpatient_508.pdf. Published 2016. Accessed October 21, 2019.

3. Spivak ES, Cosgrove SE, Srinivasan A. Measuring appropriate antimicrobial use: attempts at opening the black box. Clin Infect Dis 2016;63:1639-1644.

4. Gerber JS, Prasad PA, Fiks AG, et al. Effect of an outpatient antimicrobial stewardship intervention on broad-spectrum antibiotic prescribing by primary care pediatricians: a randomized trial. JAMA 2013;309:23452352.

5. Meeker D, Linder JA, Fox CR, et al. Effect of behavioral interventions on inappropriate antibiotic prescribing among primary care practices: a randomized clinical trial. JAMA 2016;315:562-570.

6. Allen JM, Dunn R, Bush J. Effect of prescriber peer comparison reports on fluoroquinolone use across a 16-facility community hospital system. $J$ Am Coll Clin Pharm 2019:6.

\title{
Detection of the emergence of mcr-1-mediated colistin-resistant Escherichia coli and Klebsiella pneumoniae through a hospital-based surveillance in an oncology center in eastern India
}

\author{
Samadrita Roy $\mathrm{MSc}^{1}$, Parijat Das MSc, $\mathrm{PhD}^{1}$, Surojit Das MSc, $\mathrm{PhD}^{2}$, Subhanita Roy $\mathrm{MSc}^{1}$, Soumik Pal MSc ${ }^{1}$, \\ Vinitha Mary Joy MD ${ }^{1}$, Sudipta Mukherjee MD, IDCCM, FNB, EDICM ${ }^{3}$, Arpita Bhattacharyya MRCP4, \\ Gaurav Goel MD, DNB, MNAMS ${ }^{1}$, Sanjay Bhattacharya MD, DNB, FRCPath ${ }^{1}$ (1), Purva Mathur MD ${ }^{5}$, \\ Kamini Walia PhD, $\mathrm{MPH}^{6}$ and Mammen Chandy MD, FRCPA, FRACP, FRCP \\ ${ }^{1}$ Department of Microbiology, Tata Medical Center, Kolkata, India, ${ }^{2}$ Department of Biomedical Laboratory Science and Management, Vidyasagar University, West \\ Bengal, India, ${ }^{3}$ Department of Anaesthesia \& Critical Care Medicine, Tata Medical Center, Kolkata, India, ${ }^{4}$ Department of Paediatric Hematology and Oncology, \\ Tata Medical Center, Kolkata, India, ${ }^{5}$ Department of Laboratory Medicine, JPNA Trauma Centre, All India Institute of Medical Sciences, New Delhi, India, ${ }^{6}$ Indian \\ Council of Medical Research, New Delhi, India and ${ }^{7}$ Department of Clinical Hematology, Tata Medical Center, Kolkata, India
}

Author for correspondence: Sanjay Bhattacharya, MD,

Tata Medical Center, Kolkata, India. E-mail: sanjay.bhattacharya@tmckolkata. com

Cite this article: Roy S, et al. (2020). Detection of the emergence of $m c r-1$-mediated colistin-resistant Escherichia coli and Klebsiella pneumoniae through a hospital-based surveillance in an oncology center in eastern India. Infection Control \& Hospital Epidemiology, 41: 378-380, https://doi.org/10.1017/ice.2019.363
To the Editor-The global emergence of colistin-resistant Enterobacteriaceae through the plasmid-mediated $\mathrm{mor}$ gene has raised concerns with regard to spread of antimicrobial resistance and infection control. ${ }^{1}$ There are several mechanisms of colistin resistance. ${ }^{2}$ However, the molecular epidemiology of colistin resistance in clinically relevant gram-negative isolates is poorly defined in India, a country that is large and populous and is also a major 